\title{
Calculation of Electron Swarm Parameters in Tetrafluoromethane
}

\author{
Idris H. Salih ${ }^{1}$, Mohammad M. Othman ${ }^{2}$ and Sherzad A. Taha ${ }^{2}$ \\ ${ }^{1}$ Tishk International University, \\ Erbil, Kurdistan Region - F.R. Iraq \\ ${ }^{2}$ Department of Physics, College of Education, Salahaddin University-Erbil, \\ Erbil, Kurdistan Region - F.R. Iraq
}

\begin{abstract}
The electron swarm parameters and electron energy distribution function (EEDF) are necessary, especially on understanding quantitatively plasma phenomena and ionized gases. The EEDF and electron swarm parameters including the reduce effective ionization coefficient $(\alpha-\eta) / N(\alpha$ and $\eta$ are the ionization and attachment coefficient, respectively), electron drift velocity, electron mean energy, characteristic energy, density normalized longitudinal diffusion coefficient, and density normalized electron mobility in tetrafluoromethane $\left(\mathrm{CF}_{4}\right)$ which was analyzed and calculated using the two-term approximation of the Boltzmann equation method at room temperature, over a range of the reduced electric field strength $(\mathrm{E} / \mathrm{N})$ between 0.1 and $1000 \mathrm{Td}$ $\left(1 \mathrm{Td}=10^{-17} \mathrm{~V} \cdot \mathrm{cm}^{2}\right)$, where $\mathrm{E}$ is the electric field and $\mathrm{N}$ is the gas density of the gas. The calculations required cross-sections of the electron beam, thus published momentum transfer, vibration, electronic excitation, ionization, and attachment cross-sections for $\mathrm{CF}_{4}$ were used, the results of the Boltzmann equation in a good agreement with experimental and theoretical values over the entire range of $E / N$. In all cases, negative differential conductivity regions were found. It is found that the calculated EEDF closes to Maxwellian distribution and decreases sharply at low $\mathrm{E} / \mathrm{N}$. The low energy part of EEDF flats and the high-energy tail of EEDF increases with increase $E / N$. The EEDF found to be non-Maxwellian when the $\mathrm{E} / \mathrm{N}>10 \mathrm{Td}$, having energy variations which reflect electron/molecule energy exchange processes. In addition, limiting field strength $(E / N)_{\text {limit }}$ has been calculated from the plots of $(\alpha-\eta) / N$, for which the ionization exactly balances the electron attachment, which is valid for the analysis of insulation characteristics and application to power equipment.
\end{abstract}

Index Terms-Boltzmann equation, CF4, Electron discharge, Electron swarm parameters, Kinetic and transport theory.

\section{INTRODUCTION}

Tetrafluoromethane, also known as carbon tetrafluoride or $\mathrm{R}-14$ is the simplest fluorocarbon $\left(\mathrm{CF}_{4}\right)$, was first observed

ARO-The Scientific Journal of Koya University

Vol. VIII, No.2 (2020), Article ID: ARO.10671, 7 pages

DOI:10.14500/aro.10671

Received 03 May 2020; Accepted 08 July 2020

Regular research paper: Published 01 September 2020

Corresponding author's e-mail: sherzad.taha@su.edu.krd

Copyright (C) 2020 Idris H. Salih, Mohammad M. Othman,

Sherzad A. Taha. This is an open-access article distributed under the

Creative Commons Attribution License. as an impurity in krypton by Gassman, 1974, it has a low critical temperature and high critical pressure. $\mathrm{The}^{\mathrm{CF}_{4}}$ is a man-made gas which has an important role in technological applications such as semiconductor, etching plasma for material processing, discharge opening switches, atmospheric physics and chemistry, gaseous dielectrics, and gaseous detector technology (Hunter, et al., 1985; Proshina, et al., 2015). Tetrafluoromethane is combined with oxygen as an etching medium used in the semiconductor industry for etching of dielectric materials, such as $\mathrm{SiO}_{2}$ and also for deposition of fluorinated polymer films. In combination $\mathrm{CF} 4$ with $\mathrm{SF}_{6}$ produce a less expensive, toxic, and corrosive cryogenic liquid than $\mathrm{SF}_{6}$ alone (Reinking, et al., 1986).

Tetrafluoromethane $\left(\mathrm{CF}_{4}\right)$ is a very powerful greenhouse, it is stable and does not deplete the ozone layer, which strongly absorbs infrared radiation at $\sim 8 \mu \mathrm{m}$. The lifetime of $\mathrm{CF}_{4}$ is 50,000 years, with a global warming potential (GWP) 6500 times greater than that of $\mathrm{CO}_{2}$ over a 100 -year time scale, only about $26.36 \%$ of that of $\mathrm{SF}_{6}$ (Muhle, et al., 2010; Tezcan, et al., 2016).

Tetrafluoromethane, at room temperature and atmospheric pressure, is a colorless, odorless, easy to compress gas, high stability, non-toxic, and non-flammable gas. $\mathrm{CF}_{4}$ has been used not only in the form of pure but also in mixtures with other gases for much industrial application. In high-voltage technology, $\mathrm{CF}_{4}$ is used as an admixture for circuit breakers (Duzkaya and Tezcan, 2017).

For example, binary mixtures of $\mathrm{CF}_{4}$ and $\mathrm{Ar}$ are used in microelectronics circuit applications (Cox, et al., 1989), whereas $\mathrm{CF}_{4} / \mathrm{Ar}$ mixtures have been also used in the application of capacitively coupled plasma (CCP) (Kitajima, et al., 2000) and possibly even inductively coupled plasma (Hioki, et al., 2000). The mixture of $\mathrm{SF}_{6}$ and $\mathrm{CF}_{4}$ has found applications in high-voltage circuit breakers in a cold climate which has greatly reduced the GWP compared with pure $\mathrm{SF}_{6}$ (Xiao, et al., 2004). A mixture of $50 \% \mathrm{SF}_{6}-50 \% \mathrm{CF}_{4}$ has been proposed as an insulating gas for electrical equipment, which was successfully utilized for circuit breakers (Wu, et al., 2006).

The electron swarm parameters of $\mathrm{CF}_{4}$, which are the drift velocity, characteristic energy, mean electron energy, electron mobility, diffusion coefficient ionization, attachment, and effective ionization coefficients, are widely investigated in the 
literature (Bordage, et al., 1996; Bordage and Segur, 1999; Vasenkov, 1999; Christophorou and Olthoff, 2004; Xiao and Deng, 2013; Sang-Nam, 2015; Michele, 2018). These swarm parameters are also calculated in $\mathrm{CF}_{4}-\mathrm{Ar}$ (Kurihara, et al., 2000; Tezcan, et al., 2013), $\mathrm{CF}_{4}-\mathrm{SF}_{6}$ (Xueli and Xiao, 2007), and $\mathrm{CHF}_{3}-$ $\mathrm{CF}_{4}$ gas mixtures (Duzkaya and Tezcan, 2019). Furthermore, the ternary mixtures $\mathrm{SF}_{6}-\mathrm{CF}_{4}-\mathrm{Ar}$ were also analyzed by solving Boltzmann's equation (Tezcan, et al., 2016; Tezcan, et al., 2016).

In the present study, the behavior of electron swarm parameters in pure tetrafluoromethane $\left(\mathrm{CF}_{4}\right)$ is studied and analyzed using the two-term approximation of the Boltzmann equation in the range of density reduced electric field strength $\mathrm{E} / \mathrm{N}$ varying from 0.1 to $1000 \mathrm{Td}\left(1 \mathrm{Td}=10^{-17} \mathrm{Vcm}^{2}\right)$, where $\mathrm{E}$ is the electric field and $\mathrm{N}$ is neutral number density.

\section{THEORY}

\section{A. The Boltzmann Equation}

The role of electron energy distribution function (EEDF) is an important parameter to compute the electron swarm parameters, it is difficult to measure experimentally. The EEDF calculated from a set of electron gas collision crosssection using Monte Carlo simulation method or Boltzmann equation (two-term or multi-term solution), when the electron collision frequency for inelastic collisions is much smaller than the collision frequency for elastic collision, it is the condition the two-term solution to be valid (Smith and Thomson, 1978). The steady-state distribution function is then given by the solution of the Boltzmann equation which is based on the two-term theory (Pinheiro and Loureiro, 2002). A detailed solution of Boltzmann equation used in this study to calculate electron swarm parameters may be found in the literatures (Holstein, 1946; Frost and Phelps, 1962; Smith and Thomson, 1978; Hagelaar and Pitchford, 2005). The basic of Boltzmann transport equation used in this study is given as,

$$
\begin{aligned}
& \frac{E^{2}}{3} \frac{d}{d \varepsilon}\left(\frac{\varepsilon}{N Q_{m}^{T}(\varepsilon)} \frac{d f_{0}(\varepsilon)}{d \varepsilon}\right)+\frac{2 m}{M} \frac{d}{d \varepsilon} \\
& \left(\varepsilon^{2} N Q_{m}^{T}(\varepsilon) f_{0}(\varepsilon)\right)+\frac{2 m K_{B} T_{g}}{M e}\left(\varepsilon^{2} N Q_{m}^{T}(\varepsilon) \frac{d f_{0}(\varepsilon)}{d \varepsilon}\right)+ \\
& \sum_{J}\left(\varepsilon+\varepsilon_{J}\right) f_{0}\left(\varepsilon+\varepsilon_{J}\right) N Q_{J}\left(\varepsilon+\varepsilon_{J}\right) \\
& -\varepsilon f_{0}(\varepsilon) N_{J} \sum_{J} Q_{J}(\varepsilon)=0
\end{aligned}
$$

Equation (1) is the so-called homogeneous electron Boltzmann transport equation and the isotropic component of the electron velocity distribution function, $\mathrm{f}_{\mathrm{o}}(\mathrm{v})$, can be expressed in terms of the electron energy $\varepsilon=0.5 \mathrm{mv}^{2}$, being then termed isotropic EEDF depends on the electron energy. $\mathrm{K}_{\mathrm{B}}$ is the Boltzmann constant, $\mathrm{T}_{\mathrm{g}}$ is the gas temperature, $\mathrm{m} / \mathrm{M}$ is the ratio of electronic to atomic mass, $\mathrm{Q}_{\mathrm{J}}(\varepsilon)$ is the cross-sections for transitions from the ground state to the various excited states $\mathrm{J}$, and $\varepsilon_{\mathrm{J}}$ is the threshold energy of the Jth excitation process, and $Q_{m}^{e}(\varepsilon)$ is the total effective momentum transfer cross section defined as follows,

$$
Q_{m}^{T}(\varepsilon)=Q_{m}(\varepsilon)+\sum_{j} Q_{e}(\varepsilon)+Q_{i}(\varepsilon)+Q_{a}(\varepsilon)
$$

Where, $Q_{\mathrm{m}}(\varepsilon), Q_{\mathrm{e}}(\varepsilon), Q_{\mathrm{i}}(\varepsilon)$, and $Q_{\mathrm{a}}(\varepsilon)$ are the electron cross-sections of momentum transfer, excitation, ionization, and attachment, respectively.

The $4^{\text {th }}$ term in Equation (1) expresses inelastic loss processes due to electrons of energy $\left(\varepsilon+\varepsilon_{J}\right)$ undergoing a collision in which they lose the energy $\varepsilon_{J}$ and appear as electrons of energy $\varepsilon$. The last term expresses gain of energy by electrons due to the second kind collision.

Equation (1) applies to swarm of electron drifting through gas and mixtures under the influence of a uniform dc electric field $\mathrm{E}$ in $\mathrm{V} / \mathrm{cm}$.

\section{B. Transport Parameters}

The electron transport coefficient in given gases calculated using a two-term approximation of the Boltzmann equation is functions of the density reduced electric field strength $\mathrm{E} / \mathrm{N}$, the gas temperature, and electron collision cross-section sets. The EEDF plays an important role in the calculation of electron swarm parameters.

The EEDF can be normalized by Colonna and D'Angola, 2016 ,

$$
\int_{0}^{\infty} f(\varepsilon) \sqrt{\varepsilon} d \varepsilon=1
$$

Taking into account, the normalization condition, $f_{o}(\varepsilon)$ and $f(\varepsilon)$ are linked to one another as follows,

$$
f(\varepsilon)=\frac{1}{n_{e}} \frac{4 \pi}{m} \sqrt{\frac{2}{m}} f_{o}(\varepsilon)
$$

With such normalization, the Maxwellian electron distribution function at temperature $\mathrm{T}_{\mathrm{g}}$ writes (Jiang and Economou, 1993),

$$
f(\varepsilon)=\frac{2}{\sqrt{\pi}}\left(K_{B} T_{g}\right)^{-3 / 2} \exp \left(-\frac{\varepsilon}{K_{B} T_{g}}\right)
$$

In terms of the EEDF, $f(\varepsilon)$, the mean electron energy is expressed as,

$$
\langle\varepsilon\rangle=\int_{0}^{\infty} \varepsilon^{3 / 2} f(\varepsilon) d \varepsilon
$$

With $\langle\varepsilon\rangle=\frac{3}{2} K_{B} T_{g}$, in the case of a Maxwellian, while the electron mobility $\mu_{\mathrm{e}}=\sigma_{\mathrm{e}} / \mathrm{en}_{\mathrm{e}}$, where $\sigma_{\mathrm{e}}$ the electron conductivity (Lee and More, 1984), is given by the equation,

$$
\mu_{e}=-\frac{2}{3} \frac{e}{m} \int_{0}^{\infty} \frac{\varepsilon^{3 / 2}}{v_{m}^{e}(\varepsilon)} \frac{d f}{d \varepsilon} d \varepsilon
$$

Where, $v_{m}^{e}$ is collision frequency, making the substitution $v_{m}^{e}=n_{\circ} Q_{m}^{T}$, we obtain the following expression for the reduced mobility, 


$$
N \mu_{e}=-\frac{1}{3} \sqrt{\frac{2 e}{m}} \int_{0}^{\infty} \frac{\varepsilon}{Q_{m}^{T}(\varepsilon)} \frac{\partial f(\varepsilon)}{\partial \varepsilon} d \varepsilon
$$

The electron drift velocity, diffusion coefficient, and characteristic energy are given as Smith and Thomson, 1978,

$$
v_{d}=-\frac{\bar{E}}{3} \sqrt{\frac{2 e}{m}} \int_{0}^{\infty} \frac{\varepsilon}{N Q_{m}^{T}(\varepsilon)} \frac{\partial f(\varepsilon)}{\partial \varepsilon} d \varepsilon
$$

The transverse diffusion coefficient $\mathrm{D}_{\mathrm{T}}$ is given by,

$$
\begin{gathered}
D_{T}=\frac{1}{3} \sqrt{\frac{2 e}{m}} \int_{0}^{\infty} \frac{\varepsilon}{N Q_{m}^{T}(\varepsilon)} f(\varepsilon) d \varepsilon \\
\varepsilon_{k}=\frac{e D_{T}}{\mu_{e}}
\end{gathered}
$$

From the computed drift velocity $v_{\mathrm{d}}$, the ionization and attachment coefficients are obtained as Do, 2016,

$$
\begin{aligned}
& \frac{\alpha}{N}=\frac{1}{v_{d}} \sqrt{\frac{2 e}{m}} \int_{i}^{\infty} Q_{i}(\varepsilon) f(\varepsilon) \varepsilon^{1 / 2} d \varepsilon \\
& \frac{\eta}{N}=\frac{1}{v_{d}} \sqrt{\frac{2 e}{m}} \int_{a}^{\infty} Q_{a}(\varepsilon) f(\varepsilon) \varepsilon^{1 / 2} d \varepsilon
\end{aligned}
$$

Where, $Q_{\mathrm{i}}(\varepsilon)$ and $Q_{\mathrm{a}}(\varepsilon)$ are ionization and attachment cross-section, here, $i$ and a are the ionization and attachment threshold energy. The reduced limit electric field strength $(\mathrm{E} / \mathrm{N})_{\text {limit. }}$ is calculated when the formation and loss electrons reach a balance, this means that the effective ionization equal to zero $(\alpha-\eta) / \mathrm{N}=0$ ( $\mathrm{Li}$, et al., 2012).

\section{Collision Cross-SECTION}

The EEDF and values of transport coefficients in tetrafluoromethane $\left(\mathrm{CF}_{4}\right)$ gas calculated from the sets cross-section (elastic and inelastic) reported by Kurihara, et al., 2000. This set includes 16 collision processes: One momentum transfer cross-section $\left(\mathrm{Q}_{\mathrm{m}}\right)$, three vibration excitations $\left(Q_{\mathrm{v} 1}, \mathrm{Q}_{\mathrm{v} 2}\right.$, and $\left.\mathrm{Q}_{\mathrm{v} 3}\right)$ with threshold energy $0.0 .108,0168$, and $0.077 \mathrm{eV}$, respectively, and one electronic excitation $\left(\mathrm{Q}_{\mathrm{ex}}\right)$ cross-section with threshold energy of $7.54 \mathrm{eV}$, one attachment cross-section $\left(\mathrm{Q}_{\mathrm{a}}\right)$ with threshold energy $6.4 \mathrm{eV}$, seven dissociation ionization cross-sections $\left(\mathrm{Q}_{\mathrm{i} 1}-\mathrm{Q}_{\mathrm{i}}\right)$ with threshold energy 16, 21, 26, 24, 34, 41, and $42 \mathrm{eV}$. respectively, and three neutral dissociation crosssections $\left(\mathrm{Q}_{\mathrm{d} 1}-\mathrm{Q}_{\mathrm{d} 3}\right)$ with threshold energy 12,17 , and $18 \mathrm{eV}$, respectively.

\section{Results AND Discussion}

The EEDF and electron swarm parameters in pure tetrafluoromethane $\left(\mathrm{CF}_{4}\right)$ are calculated using the twoterm approximation of Boltzmann equation in dc uniform fields in the range $0.1-1000 \mathrm{Td}$, at 1 Torr and $300 \mathrm{~K}$.
Tetrafluoromethane gas has the momentum transfer cross-section which is greater than inelastic cross-section, this is a necessary condition for the two-term approximation solution of the Boltzmann equation to be valid (Smith and Thomson, 1978). The EEDF is one of the most important parameters for gas discharge phenomena and used for calculating electron swarm parameters. The $\mathrm{E} / \mathrm{N}$ values were chosen to yield mean electron energies in the range of $0.045-14.3 \mathrm{eV}$. The EEDF for a dc field with various $\mathrm{E} / \mathrm{N}$ values is given in Fig. 1 (where, $\mathrm{E}$ is the electric field and $\mathrm{N}$ is the gas density).

The influence of the EEDF versus the mean electron energy for different ratios of electric field strength $(\mathrm{E} / \mathrm{N})$ is shown in Fig. 1. The EEDF is strongly affected by changing parameter $\mathrm{E} / \mathrm{N}$, the higher values of $\mathrm{E} / \mathrm{N}$ lead to the extension of the EEDF curves toward higher energy tail. The distribution function is normalized by Equation (3), the outcome of EEDF curves is due to constant electron density $\mathrm{n}_{\mathrm{e}}=1 \times 10^{16} \mathrm{~cm}^{-3}$. For the lowest $\mathrm{E} / \mathrm{N} \leq 1 \mathrm{Td}$, where $1 \mathrm{Td}=10^{-17} \mathrm{~V} \cdot \mathrm{cm}^{2}$. The electron energy is thermal and the ionization degrees $\left(\mathrm{n}_{\mathrm{e}} / \mathrm{N}\right)>\left(\mathrm{Q} / 10^{-13}\right) \varepsilon^{2}$ (where, $\varepsilon$ is energy in $\mathrm{eV}$ and $\mathrm{Q}$ is an elastic and inelastic cross-section in the unit of $\mathrm{cm}^{2}$ ) of the order of $10^{-3}-10^{-4}$ can be sufficient to give Maxwellian characteristics of the EEDF as indicated by the straight line with a slope of $\left(-1 / \mathrm{K}_{\mathrm{B}} \mathrm{T}_{\mathrm{g}}\right)$. In this case, the ionization degree is very small and the tetrafluoromethane $\left(\mathrm{CF}_{4}\right)$ atoms are in the ground state. However, in the range $\mathrm{E} / \mathrm{N}>1 \mathrm{Td}$, the distribution is non-Maxwellian, the tail of the EEDF falls off rapidly as high-energy electrons are depleted due to different inelastic collisions. This behavior can be attributed to the electric field that heats the electrons and the energy of cold electrons increases. Subsequently, the mean electron energy depends on the electric field strength $\mathrm{E} / \mathrm{N}$.

Using EEDF, the electron swarm parameters have been calculated. Fig. 2 shows our calculated results for electron drift velocity as a function of $\mathrm{E} / \mathrm{N}$ in comparison with the corresponding results provided by previous theoretical and experimental literatures.

The agreement between the present calculation and theoretical values (Bordage and Segure, 1999; Wu, et al., 2006; Duzkaya and Tezcan, 2019) and experimental values

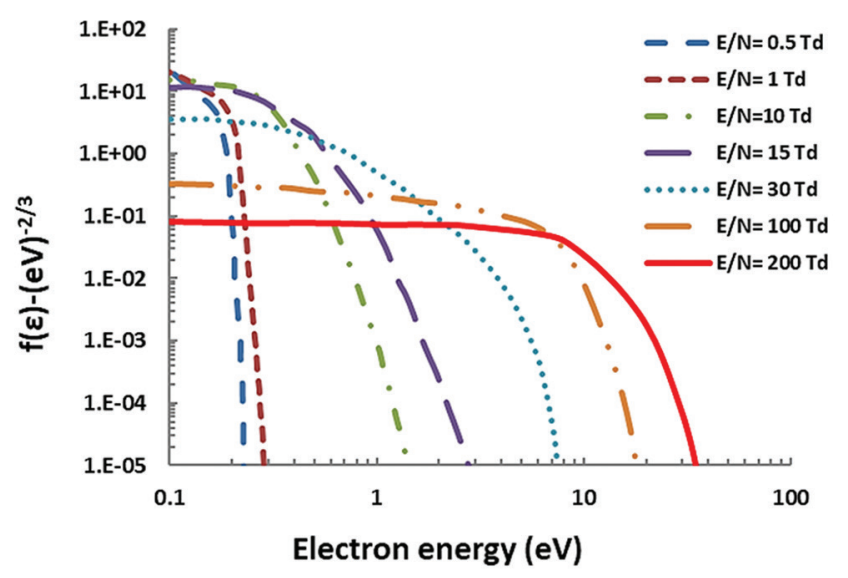

Fig. 1. Electron energy distribution functions in pure $\mathrm{CF}_{4}$ for various $\mathrm{E} / \mathrm{N}$ values. 
(Hunter, et al., 1988; Nakamura, 1996; Xiao and Deng, 2013; Duzkaya and Tezcan, 2019) is excellent. The theoretical results of Sang-Nam, 2011, in range E/N >4 $\mathrm{Td}$ are greater than present results and theoretical results of Michele, 2018, in the range $\mathrm{E} / \mathrm{N} \geq 50 \mathrm{Td}$ greater than present calculation. The drift velocity in pure CF4 showed negative differential conductivity (NDC) over the range from 15 to $60 \mathrm{Td}$, the decrease in the drift velocity was not smooth but contained a small hump. A combination of the elastic and inelastic cross-sections can occur in a tetrafluoromethane $\left(\mathrm{CF}_{4}\right)$ molecules, at low electric field strength $\mathrm{E} / \mathrm{N} \leq 20 \mathrm{Td}$ the drift velocity rapidly increases, and at the region of higher $\mathrm{E} / \mathrm{N}$ values $(20 \leq \mathrm{E} / \mathrm{N} \leq 100 \mathrm{Td})$, the drift velocity will decrease with increasing $\mathrm{E} / \mathrm{N}$ values. NDC will occur over a range of electric field strength $\mathrm{E} / \mathrm{N}$ values in $\mathrm{CF}_{4}$ when the inelastic cross-section has a large resonant type with respect to energies, and momentum transfer cross-section rapidly increases with electron energy.

Fig. 3 shows mean electron energy which increases with increasing $\mathrm{E} / \mathrm{N}$ values, it is seen that the present calculation

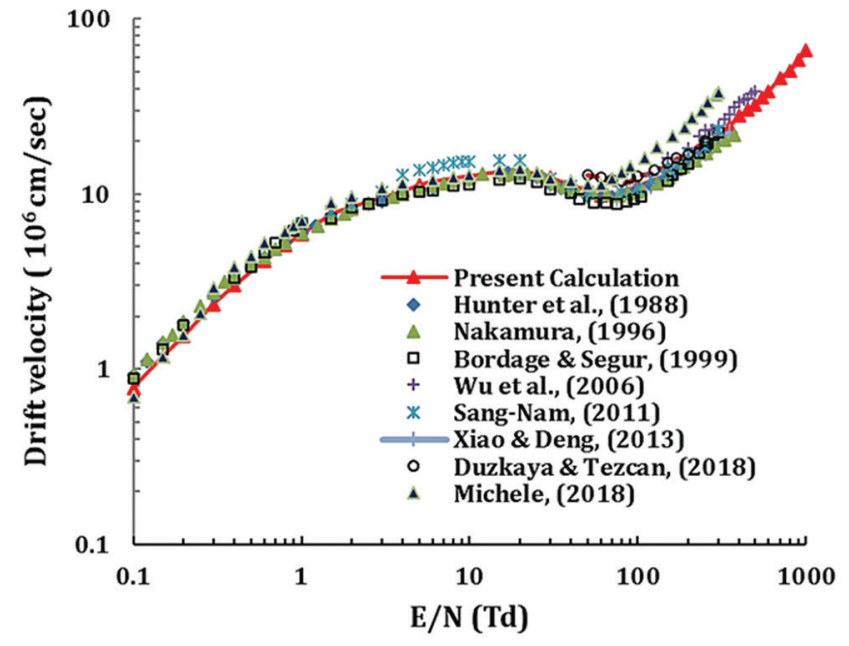

Fig. 2. Electrons drift velocity in pure $\mathrm{CF}_{4}$.

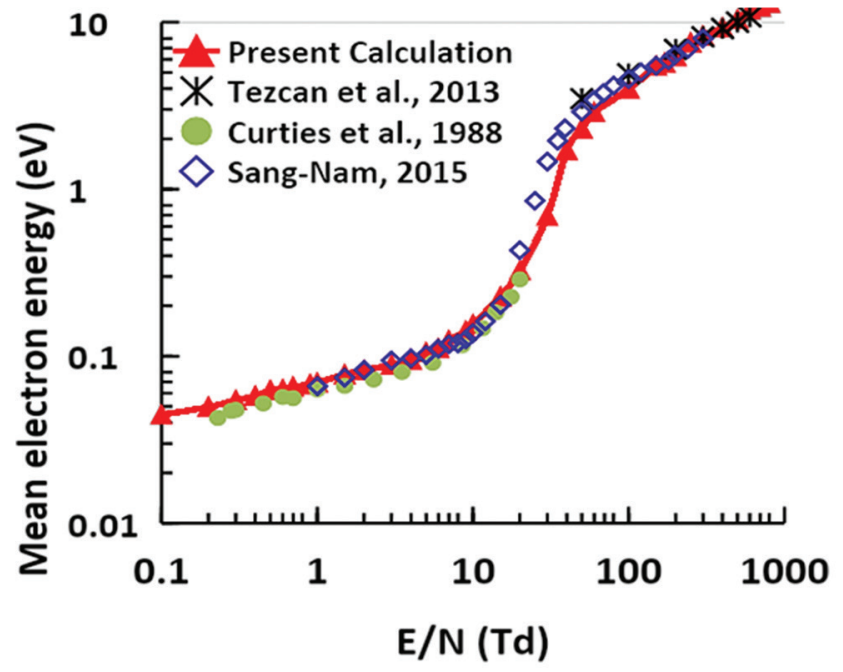

Fig. 3. Electron means energy in pure $\mathrm{CF}_{4}$. agree well with the experimental values of Curtis, et al. 1998, and theoretical values of Kurihara, et al., 2000; Tezcan, et al., 2013; Sang-Nam, 2015.

The calculated values of the characteristic energy of electrons as a function of $\mathrm{E} / \mathrm{N}$ in pure $\mathrm{CF}_{4}$ are shown in Fig. 4.

The calculated values compared with experimental values of Curtis, et al., 1988, and theoretical values of Mašek, et al., 1987; Stefanov and Pirgov, 1993; Bordage and Segur, 1999, a very good agreement was obtained, whereas the experimental values of Christophorou and Olthoff, 2004, are lower than calculated values over the $\mathrm{E} / \mathrm{N}$ range between 2 and $300 \mathrm{Td}$. The characteristic energy increases with the increase of $\mathrm{E} / \mathrm{N}$ during the inelastic collision process $(8 \mathrm{Td} \leq \mathrm{E} / \mathrm{N} \leq 80 \mathrm{Td})$ and then starts to be approximately constant at $\mathrm{E} / \mathrm{N} \geq 80 \mathrm{Td}$, where above this value, the ionization process occurs for $\mathrm{CF}_{4}$.

Fig. 5 shows density normalized electron mobility $\mu \mathrm{N}$, which decreases with increasing $\mathrm{E} / \mathrm{N}$ value, it is seen that the present calculation agree well with the experimental values of Hunter, et al., 1988.

The product of the longitudinal diffusion coefficient and the number density $\mathrm{ND}_{\mathrm{L}}(\mathrm{E} / \mathrm{N})$ is shown in Fig. 6. The results

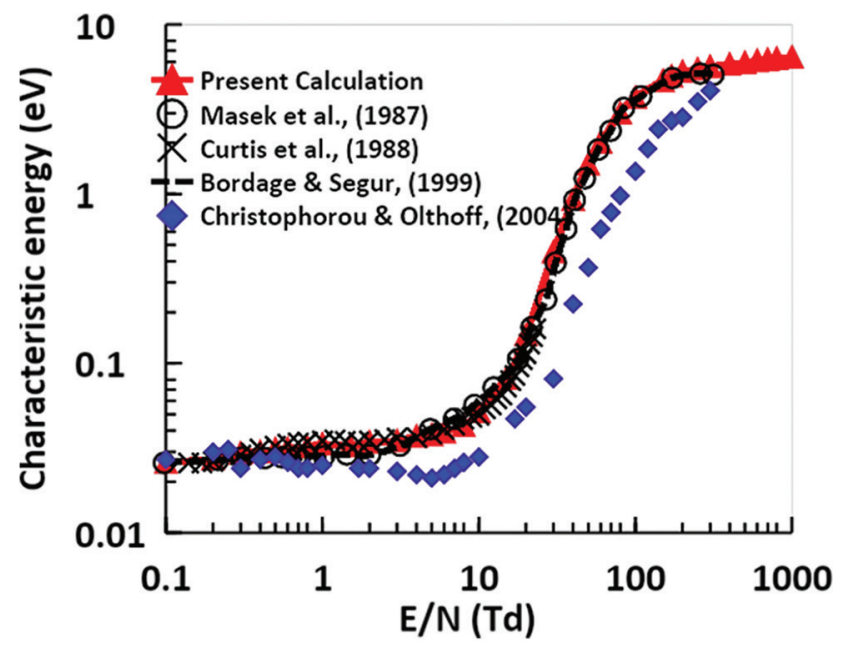

Fig. 4. Characteristic energy in pure $\mathrm{CF}_{4}$.

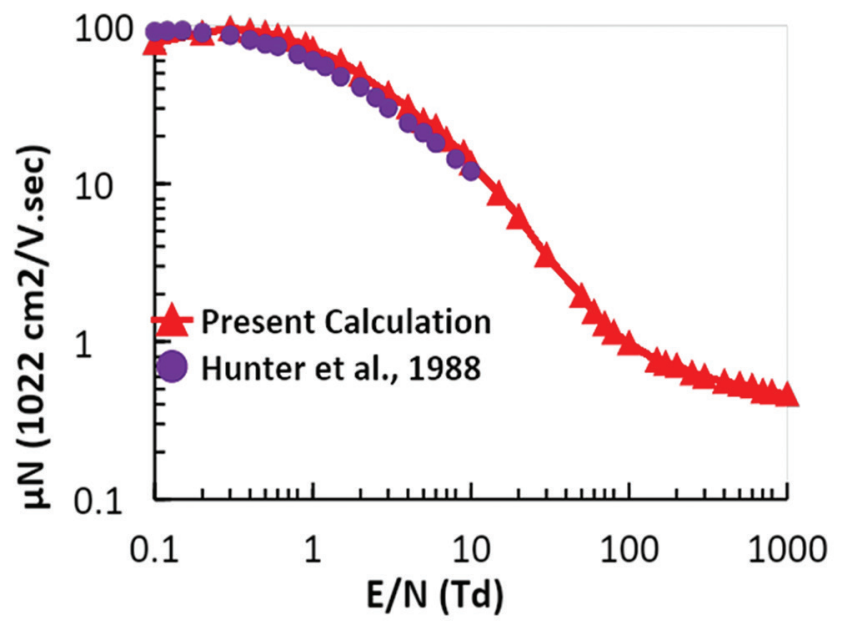

Fig. 5. Density normalized electron mobility $\mu \mathrm{N}$ in pure $\mathrm{CF}_{4}$. 
compared with the experimental values of Nakamura, 1996; Christophorou and Olthoff, 2004, and theoretical values of Kurihara, et al., 2000; Wu, et al., 2006.

The agreement is excellent over much of the range of $\mathrm{E} / \mathrm{N}$, with discrepancies in magnitude up to $10 \%$ in the region between 150 and $500 \mathrm{Td}$ between present calculation and experimental values of Xiao and Deng, 2013. The density normalized ionization coefficient $\alpha / \mathrm{N}$ has been calculated for the range $80 \leq \mathrm{E} / \mathrm{N} \leq 1000 \mathrm{Td}$ using Equation (12) and the results are shown in Fig. 7.

A good agreement has been obtained with the theoretical values of Mašek, et al., 1987; Bordage et al., 1999; SanNam, 2012, and experimental values of Hunter, et al., 1988, for the whole range of E/N. Fig. 8 shows the density normalized attachment coefficient $\eta / \mathrm{N}$ as a function of $\mathrm{E} / \mathrm{N}$. Good agreement has been obtained with theoretical values of Bordage and Segur, 1999, and experimental values of Hunter, et al., 1988. The agreement between present data and theoretical values of San-Nam, 2012, reasonably good

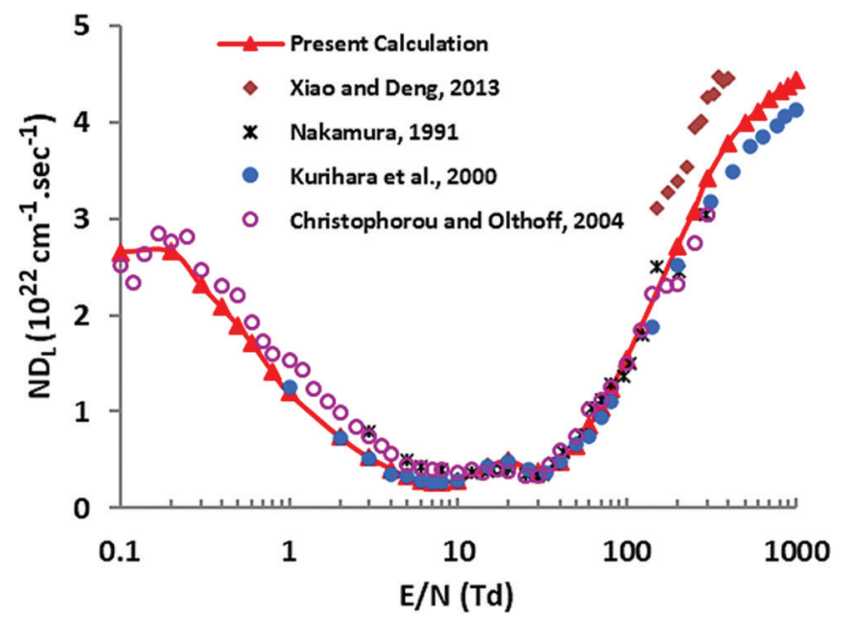

Fig. 6. The product of the longitudinal diffusion coefficient and the gas number density in pure $\mathrm{CF}_{4}$.

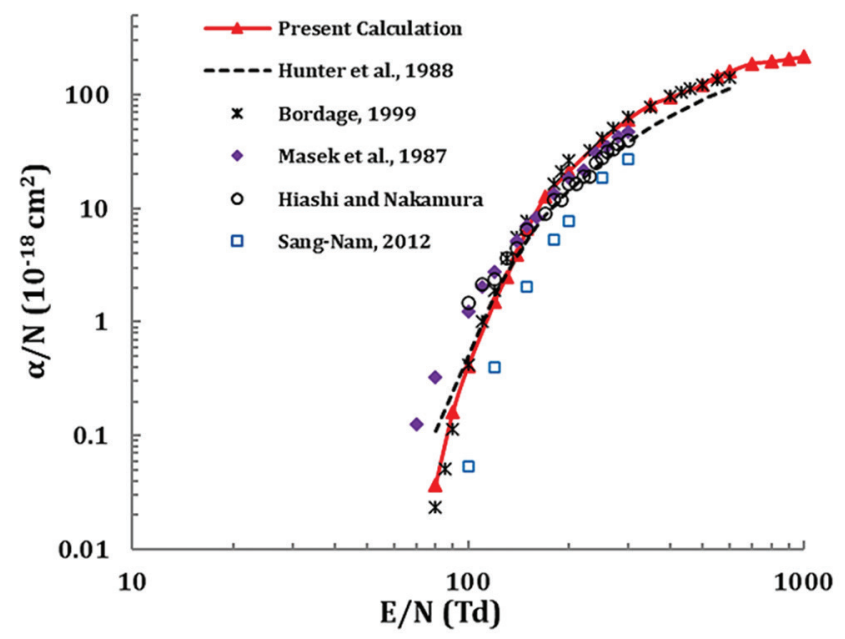

Fig. 7. Ionization coefficient in pure $\mathrm{CF}_{4}$. over the entire range of $\mathrm{E} / \mathrm{N}$, with the largest discrepancies occurring between 120 and $300 \mathrm{Td}$.

The reduced limiting electric field strength $(\mathrm{E} / \mathrm{N})_{\text {limit }}$ is an important quantity calculated from ionization and attachment coefficients. It is the value of the density reduced electric field at which $(\alpha-\eta) / \mathrm{N}=0$. This value should also be equal to the breakdown voltage of $\mathrm{CF}_{4}$ as calculated under the uniform field, this parameter is important for some high-voltage applications. The density normalized effective ionization coefficients $(\alpha-\eta) / \mathrm{N}=0$ in pure $\mathrm{CF}_{4}$ are shown in Fig. 9.

The change in the attachment coefficient is more obvious than the change in the ionization coefficient, so the change of the effective ionization coefficient is more depending on the change in the attachment coefficient. The present limiting electric field strength $(\mathrm{E} / \mathrm{N})_{\text {limit }}$ of pure $\mathrm{CF}_{4}$ is evaluated as $146 \mathrm{Td}$, which is comparable with experimental values reported by Christophorou and Olthoff, 2004, and theoretical values of Liu and Xiao, 2007; Duzkaya and Tezcan, 2019, where the estimated values of $(\mathrm{E} / \mathrm{N})_{\text {limit }}$ are 146,144 , and $145 \mathrm{Td}$, respectively.

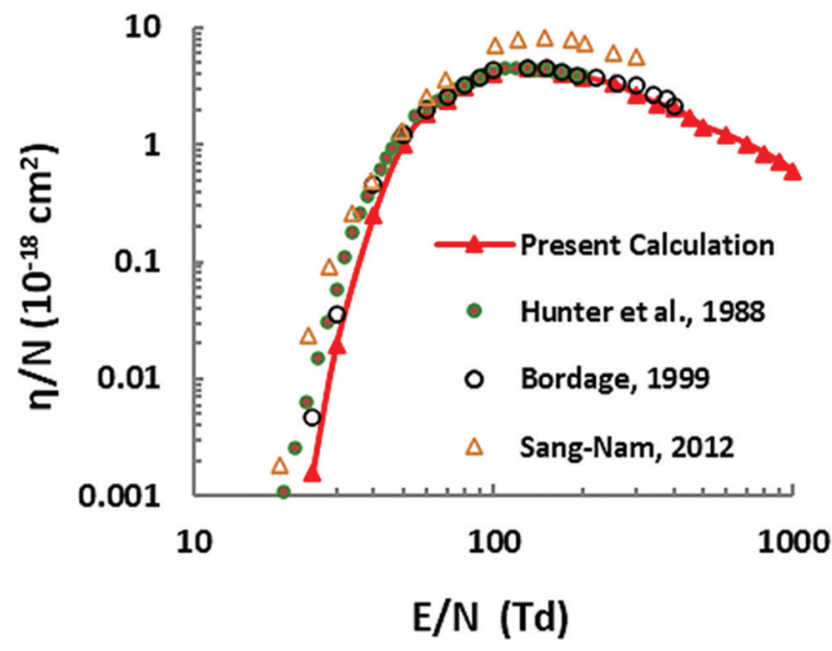

Fig. 8. Attachment coefficient in pure $\mathrm{CF}_{4}$.

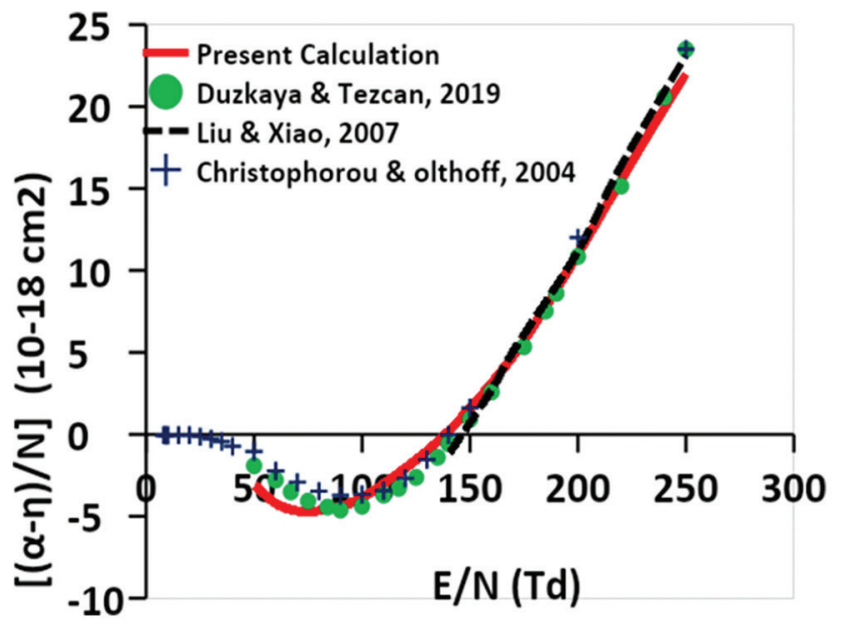

Fig. 9. Effective ionization coefficient $(\alpha-\eta) / \mathrm{N}$ in pure $\mathrm{CF}_{4}$. 


\section{CONCLUSION}

Tetrafluoromethane $\left(\mathrm{CF}_{4}\right)$ is an electronegative and potent greenhouse gas. In this study, the EEDF, electron swarm parameters, and density normalized effective ionization coefficient of tetrafluoromethane $\left(\mathrm{CF}_{4}\right)$ are calculated using the two-term approximation solution of Boltzmann equation method for the range reduced electrical field strength varying from $0.1 \mathrm{Td}$ to $1000 \mathrm{Td}$ at temperature $300 \mathrm{~K}$. The present results based on electron collision cross-section sets for $\mathrm{CF}_{4}$ molecules. The EEDF is strongly affected by changing the parameter $\mathrm{E} / \mathrm{N}$, we have shown that the EEDF in pure $\mathrm{CF}_{4}$ shifts toward increasing of mean electron energy according to the increasing of $\mathrm{E} / \mathrm{N}$. The calculated swarm parameters (drift velocity, characteristic energy, mean electron energy, diffusion coefficient, electron mobility, ionization, and attachment coefficient) agree well with experimental and theoretical values. The reduced limiting electric field strength $(\mathrm{E} / \mathrm{N})_{\text {limit }}$, at which the ionization and attachment rate are exactly balanced as a function of $\mathrm{E} / \mathrm{N}$, has been calculated from the effective ionization curves.

\section{REFERENCES}

bordage, M.C., and Segur, P., 1999. Boltzmann analysis of electron swarm parameters in $\mathrm{CF}_{4}$ using independently assessed electron-collision cross sections. Journal of Applied Physics, 86(7), pp.3558-3566.

Bordage, M.C., Segur, P., and Chouki, A., 1996. Determination of a set of electron impact cross sections in tetrafluoromethane consistent with experimental determination of swarm parameters. Journal Applied Physics, 80(3), pp. 13251336 .

Christophorou, L.G., and Olthoff, J.K., 2004. Fundamental Electron Interactions with Plasma Processing Gases. Kluwer Academic/Plenum Publisher, New York, pp.135-218.

Colonna, G., and D'Angelo A., 2016. The Two-term Boltzmann Equation. Plasma Modeling Methods and Applications. Ch. 2., IOP Publishing Ltd., United Kingdom, pp.2-34.

Cox, T.I., Deshmukh, V.G.I., and Armour, D.G., 1989. Reactive ion beam etching studies of tungsten with CF4/argon mixtures using ion scattering spectroscopy and SIMS. Vacuum, 39(11-12), pp.1171-1173.

Curtis, M.G., Isobel, C.W., and Mathieson, K.J., 1988. Electron swarm characteristic energies $\left(\mathrm{D}_{\mathrm{r}} / \mu\right)$ in tetrafluoromethane $\left(\mathrm{CF}_{4}\right)$ at low E/N. Journal of Physics D: Applied Physics, 21(8), pp.1271-1274.

Do, A.T., 2016. Analysis of electron transport coefficients in binary mixtures of TEOS gas with $\mathrm{Kr}, \mathrm{Xe}, \mathrm{He}$, and $\mathrm{Ne}$ gases for using in plasma assisted thin film deposition. Journal of Electrical Engineering and Technology, 11(2), pp.455-462.

Duzkaya, H., and Tezcan, S.S., 2017. Measurement and calculation of breakdown voltage in $\mathrm{CF}_{4}$ gas mixtures. Gazi Üniversitesi Journal Science Part C, 5(3), pp.185-195.

Duzkaya, H., and Tezcan, S.S., 2019. Boltzmann analysis of electron swarm parameters in $\mathrm{CHF}_{3}+\mathrm{CF}_{4}$ mixtures. Turkish Journal of Electrical Engineering and Computer Sciences, 27, pp.615-622.

Frost, L.S., and Phelps, A.V., 1962. Rotational excitation and momentum transfer cross sections for electrons in $\mathrm{H}_{2}$ and $\mathrm{N}_{2}$ from transfer coefficients. Physical Review, 127(5), pp.1621-1633.

Gassman, M., 1974. Freon-14 in high-grade krypton and in the atmosphere. Geophysics Research Letter, 6, pp.609-612.

Hagelaar, G.J.M., and Pitchford, L.C., 2005. Solving the Boltzmann equation to obtain electron transport coefficients and rate coefficients for fluid models. Plasma Sources Science and Technology, 14(4), pp.722-733.

Hioki, K., Hirata, H., Nakano, N., Petrovic, Z.L., and Makabe, T., 2000. Diagnostics of an inductively coupled $\mathrm{CF}_{4} /$ Ar plasma. Journal of Vacuum Science Technology A Vacuum Surfaces and Films, 18(3), pp.864-872.

Holstein, T., 1946. Energy distribution of electrons in high frequency gas discharges. Physical Review, 70(5-6), pp.367-384.

Hunter, S.E., Carter, J.G., and Christophorou, L.G., 1988. Electron motion in the gases $\mathrm{CF}_{4}, \mathrm{C}_{3} \mathrm{~F}_{8}$ and $\mathrm{n}_{-} \mathrm{C}_{4} \mathrm{~F}_{10}$. Physical Review A General Physics, 38(1), pp.58-69.

Hunter, S.R., Carter, J.G., and Christophorou, L.G., 1985. Electron transport studies of gas mixtures for use in e-beam controlled diffuse discharge switches. Journal Applied Physics, 58(8), pp.3001-3015.

Jiang, P., and Economou, D.J., 1993. Temporal evolution of the electron energy distribution functions in oxygen and chlorine gases under de and ac fields. Journal of Applied Physics, 73(12), pp.8151-8160.

Kitajima, T., Takeo, Y., Petrovic, Z.L., and Makabe, T., 2000. Functional separation of biasing and sustaining voltages in two-frequency capacitively coupled plasma. Applied Physics Letters, 77(4), pp.489-491.

Kurihara, M., Petrovic, Z.L., and Makabe, T., 2000. Transport coefficients and scattering cross-sections for plasma modeling in CF4-Ar mixtures: A swarm analysis. Journal of Physics D: Applied Physics, 33(7), pp.2146-2153.

Lee, Y.T., and More, R.M., 1984. An electron conductivity model for dense plasmas. Physics of Fluids, 27(5), pp.1273-1286.

Li, X., Zhao, H., and Jia, S., 2012. Dielectric breakdown properties of $\mathrm{SF}_{6}-\mathrm{N}_{2}$ mixtures in the temperature range 300-3000K. Journal Physics D: Applied Physics, 45(44), p.445202.

Liu, X., and Xiao, D., 2007. Monte Carlo simulation of electron swarm parameters in the $\mathrm{SF}_{6} / \mathrm{CF}_{4}$ gas mixtures. The Japan Society of Applied Physics. 46(4A), pp.1664-1667.

Mašek, K., Láska, L., D’Agostino, R., and Cramarossa, F., 1987. Boltzmann equation analysis of the electron gas in CF 4 discharges plasma. Contributions to Plasma Physics, 27(1), pp.15-21.

Michele, R., 2018. A Monte-Carlo software tools for the characterization of gas mixtures in various detector. Romanian Journal of Physics, 63, pp.8-10.

Muhle, J., Ganesan, A.L., Miller, B.R., Salameh, P.K., Harth, C.M., Greally, B.R., Rigby, M., Porter, L.W., Steele, L.P., Trudinger, C.M., Krummel, P.B., O'Doherty, S., Fraser, P.J., Simmonds, P.G., Prinn, R.G., and Weiss, R.F., 2010. Perfluorocarbons in the global atmosphere: Tetrafluoromethane, hexafluoroethane, and octafluoropropane. Atmospheric Chemistry and Physics, 10(11), pp.5145-5164.

Nakamura, Y., 1996. Electron Swarm Parameters in Pure $\mathrm{CF}_{4}$ and its Electron Collision Cross Sections. Imaging Detectors in High Energy, Astroparticle and Medical Physics: Proceedings of the UCLA International Conference. World Scientific Publishing Co Inc., Singapore, pp.75-79.

Pinheiro, M.J., and Loureiro, J., 2002. Effective ionization coefficients and electron drift velocities in gas mixtures of SF6 with $\mathrm{He}, \mathrm{Xe}, \mathrm{CO}_{2}$ and $\mathrm{N}_{2}$ from Boltzmann analysis. Journal of Physics D: Applied Physics, 35(23), pp.30773084.

Proshina, O.V., Rakhimova, T.V., Lopaev, D.V., Samara, V., Baklanov, M.R., and de Marneff, J.F., 2015. Experimental and theoretical study of RF capacitively coupled plasma in Ar-CF4-CF3I mixtures. Plasma Sources Science and Technology, 25, p.055006.

Reinking, G.F., Christophorou, L.G., and Hunter, S.R., 1986. Studies of total ionization in gases/mixtures of interest to pulsed power applications. Journal Applied Physics, 60(2), pp.499-508.

Sang-Nam, K., 2011. The drift velocity of electron in $\mathrm{CF}_{4}, \mathrm{CH}_{4}, \mathrm{Ar}$ mixtures gas. The Transactions of the Korean Institute of Electrical Engineers P, 60(3), pp.105-109. 
Sang-Nam, K., 2012. Ionization and attachment coefficients in $\mathrm{CF}_{4}, \mathrm{CH}_{4}, \mathrm{Ar}$ mixtures gas. The Transactions of the Korean Institute of Electrical Engineers $P, 61(1)$, pp.13-17.

Sang-Nam, K., 2015. Electron mean energy in $\mathrm{CF}_{4}, \mathrm{CH}_{4}, \mathrm{Ar}$ mixtures. The Transactions of the Korean Institute of Electrical Engineers P, 64(4), pp.241-245.

Smith, K., and Thomson, R.M., 1978. Computer Modeling of Gas Lasers. Plenum Press, New York.

Stefanov, B., and Pirgov, P., 1993. Semiempirical method for extracting electron molecule cross sections from experimental data: $\mathrm{CF}_{4}$ as an example. Plasma Chemistry and Plasma Processing, 13(4), pp.655-671.

Tezcan, S.S., Dincer, M.S. and Bektas, S., 2016. Effective ionization coefficients, limiting electric fields, and electron energy distributions in $\mathrm{CF}_{3} \mathrm{I}_{+} \mathrm{CF}_{4}+\mathrm{Ar}$ ternary gas mixtures. Physics of Plasma, 23(7), p.073507.

Tezcan, S.S., Dincer, M.S., Bektas, S., and Hiziroglu, H.R., 2013. Boltzmann analysis of electron swarm parameters in binary $\mathrm{CF} 4+\mathrm{Ar}$ mixtures. IEEE Transactions on Dielectrics and Electrical Insulation, 20(1), pp.98-103.
Tezcan, S.S., Duzkaya, H., Dincer, M.S., and Hiziroglu, H.R., 2016. Assessment of electron swarm parameters and limiting electric fields in $\mathrm{SF}_{6}+\mathrm{CF}_{4}+\mathrm{Ar}$ gas mixtures. IEEE Transactions on Dielectrics and Electrical Insulation, 23(4), pp.1996-2005.

Vasenkov, A.V., 1999. Electron swarm parameters in carbon tetrafluoride. Journal of Applied Physics, 85(2), pp.1222-1224.

Wu, B.T., Xiao, D.M., and Zhang, L.C., 2006. Electron swarm coefficients in $\mathrm{SF}_{6}$ and $\mathrm{CF}_{4}$ gas mixtures from Monte Carlo method. The European Physical Journal Applied Physics, 35(1), pp.57-60.

Xiao, D.M., and Deng, Y., 2013. Determination of electron swarm parameters in pure $\mathrm{CHF}_{3}$ and $\mathrm{CF}_{4}$ by a time-resolved method. Plasma Science and Technology, $15(1)$, pp. $25-29$.

Xiao, D.M., Yang, J.L., and Xu, X., 2004. Electron swarm parameters in SF ${ }_{6}$ and $\mathrm{CF}_{4}$ gas mixtures. Japanese Journal of Applied Physics, 43(3A), pp.L369-L371.

Xueli, L., and Xiao, D.M., 2007. Monte Carlo simulation of electron swarm parameters in the $\mathrm{SF}_{6} / \mathrm{CF}_{4}$ gas mixtures. Japanese Journal of Applied Physics, 46(4A), pp.1663-1667. 